\author{
Wen-Jing Liu \\ Wen-Yu Hu \\ Yie-Fong Chiu \\ Tai-Yuan Chiu \\ Bee-Hong Lue \\ Ching-Yu Chen \\ Susumn Wakai
}

\section{Factors that influence physicians in providing palliative care in rural communities in Taiwan}

Received: 19 October 2004

Accepted: 12 January 2005

Published online: 22 February 2005

(C) Springer-Verlag 2005
W.-J. Liu · T.-Y. Chiu $(\bowtie) \cdot$ B.-H. Lue

C.-Y. Chen

Department of Family Medicine, College of Medicine and Hospital, National Taiwan University,

No. 7, Chung-Shan South Rd,

Taipei, Taiwan

e-mail: tychiu@ha.mc.ntu.edu.tw

Tel.: +886-2-23562878

Fax: +886-2-23710937

W.-Y. Hu

School of Nursing,

College of Medicine and Hospital,

National Taiwan University,

Taipei, Taiwan

Y.-F. Chiu

Chiu's Family Practice Center,

Tainan, Taiwan

T.-Y. Chiu · S. Wakai

Department of International

Community Health,

Graduate School of Medicine,

The University of Tokyo,

Tokyo, Japan

\begin{abstract}
Goals of work: To identify the willingness, influencing factors, and educational needs of community physicians in providing palliative care in the rural areas of Taiwan.

Methods: A questionnaire was sent to all medical directors of the 140 government health stations assigned to the rural areas of Taiwan.

Results: The overall response rate was $62.8 \%$ with 85 valid questionnaires retrieved. The majority of respondents $(84.7 \%)$ expressed a willingness to provide palliative care if they encountered an advanced cancer patient. However, they would limit their services to consultation and referral $(93.0 \%$ and $87.5 \%$, respectively), and were less likely to provide home visits $(40.3 \%)$ or bereavement support of the family (29.2\%). With respect to knowledge, the accurate answers to the philosophy/principles and clinical practice of palliative care were $93.4 \%$ and $57.3 \%$, respectively. Regarding attitudes, the highest score item in perceiving the threat about providing palliative care was "uncomfortable to meet and take care of the advanced cancer patient." The highest score item in perceiving
\end{abstract}

barriers was "providing palliative care may shorten patient's life, just like euthanasia." The results of stepwise logistic regression analysis for the willingness to provide home visits showed that only the subjective norms remained in the model $(\mathrm{OR}=1.87$, 95\% CI $=1.17-3.01)$. Educational needs expressed by the respondents were ranked as follows: emotional support to, communication skills with, and bereavement support for the advanced cancer patients and their relatives. Conclusions: Effective training courses that emphasize the practical knowledge of palliative care for community physicians, incorporating palliative care into medical education particularly in terms of communication skills and ethical roles, and active health policy administration including insurance payments, are important for the enhancement of community palliative care in Taiwan.

Keywords Palliative care .

Community $\cdot$ Knowledge $\cdot$ Belief . Willingness

\section{Introduction}

Previous studies showed that over half of advanced cancer patients prefer to stay at home and die there $[1,5,6,18$, 27]. Moreover, some claimed that home-based services are cheaper than and as effective as hospital services [2, 12-14]. In Taiwan, a community study showed that not quite half (44.6\%) of the Taiwanese population chose the home as the preferred place for terminal care. Yet nearly $60 \%$ $(59.4 \%)$ of these people, who originally chose the hospital, 
would change their mind and choose the home as the preferred place for terminal care if home palliative care was available. In that study, we also found that $80 \%$ of people hoped to die at home [7]. Another study, in 1996, showed that of 329 cancer patients in a palliative care unit, $43.1 \%$ preferred to die in the hospital and $56.9 \%$ would rather have died at home. Of these, $59.1 \%$ eventually died in the hospital and $40.9 \%$ at home. Many of those who died at home were actually kept alive just long enough to transfer from the hospital to their homes and then died soon thereafter [8]. In other studies, the majority $(80 \%)$ of primary caregivers of advanced cancer patients receiving domiciliary palliative care expressed the need for palliative care. Otherwise, the patients were highly satisfied with the home care provided by the health professionals $[15,16]$. From the above findings, we found that strengthening the competence of palliative care (in particular, home visits) in the community in Taiwan is essential.

About 35,000 people died of cancer in Taiwan in 2003 compared to 24,000 in 1994 . Over the last decade, the hospice and palliative care services have expanded in Taiwan, and there were 21 inpatients services and 45 home palliative care programs in 2003 [10]. Although the government in Taiwan has plans to extend home care to rural communities, home health care - especially for advanced cancer patients - is presently only available in some large cities $[9,10,19]$. To promote the adequacy of community palliative care, a good strategy is to build a system of home palliative care in rural areas through the physicians and their medical teams in established government health stations. To assess the feasibility of this strategy, this study chose to examine the willingness of physicians at health stations to provide palliative care in their communities and the factors that influence them.

Our study had the following objectives: (1) to identify the willingness of physicians at the health stations to provide palliative care in their communities when they encounter an advanced cancer patient, (2) to investigate the influencing factors of this willingness to provide palliative care, and (3) to determine the educational needs of these physicians in providing palliative care. With these findings, we can design effective interventions for physicians at health stations to promote community palliative care.

\section{Materials and methods}

\section{Subjects}

This study, designed with a cross-sectional survey using the cluster sampling method, was conducted in the period 2000-2002. A structured questionnaire was mailed to 140 medical directors (all physicians) at the 140 government health stations that cover most of the rural communities in Taiwan. There were a total of 174 health stations in the rural communities of Taiwan, but 34 (19.5\%) did not have physicians. Two months after the questionnaire was mailed, nonresponders were contacted again, and the questionnaire survey was resent twice. All responses received before February 1, 2002, was included in the analysis. Altogether, 88 respondents $(62.8 \%)$ returned the questionnaires. Deducting incomplete questionnaires, 85 valid questionnaires were retrieved. Participant selection and study design were approved by the National Science Council of Taiwan and the Governmental Health Bureaus in each county. All participants enrolled gave written informed consent.

\section{Measurement}

A structured self-report questionnaire consisting of six parts was administered to all subjects. The six parts of the questionnaire included questions on demographic characteristics, knowledge in palliative care, beliefs on palliative care, subjective norms, willingness, and educational needs to provide palliative care. The entire six-part questionnaire was tested for content validity by a panel comprised of two physicians, two nurses, one psychologist, and one social worker, all of whom were experienced in the care of the terminally ill. Each item in the questionnaire was appraised from "very inappropriate and not relevant" (1) to "very appropriate and relevant" (5). A "content validity index" (CVI) was used to determine the validity of the structured questionnaire. The questionnaire yielded a CVI of 0.920 computed by summing 0.238 from the "4" ratings and 0.682 from the " 5 " ratings on all items. In addition, ten family physicians filled out the questionnaire to confirm its face validity and ease of application.

Demographic characteristics assessed by the questionnaire included age, experience in providing palliative care, and information on palliative care. The other five parts included:

1. Knowledge in palliative care (Appendix A). This measure consisted of the philosophy and principles of hospice and palliative care (14 items) and practical knowledge about palliative care (14 items) such as symptom management and psychological and spiritual care to be given to individuals and their families. This 28-item measure was designed with careful scrutiny of the literature in this area. The main reference was the PCQN originally developed by Ross et al. [24, 25]. All items were also grounded on the basis of real-life experiences of the investigators involved in palliative care. The scoring system of this scale is "true" (1) and "false/unknown" (0). A Kuder-Richardson formula 20 (KR-20) was used to assess internal consistency of this knowledge measure and showed a coefficient of 0.71 .

2. Beliefs on palliative care (Appendix B). This part included the perception of the threats, benefits, and barriers in providing palliative care, designed with careful scrutiny of the literature by the investigators 
[23]. The measure is a 26-item set using a five-point Likert scale from "strongly disagree" (1) to "strongly agree" (5). Bartlett's test of sphericity and the KaiserMeyer-Olkin (KMO) test were used to determine that beliefs data was suitable for exploratory factor analysis. Therefore, the draft items were analyzed using principal component factor analysis followed by orthogonal varimax rotation. Finally, the beliefs measure was constructed using threats (six items), benefits of life quality (seven items), benefits of good death (five items), and barriers (eight items) perceived from the physicians in taking care of advanced cancer patients. Internal consistency is demonstrated with the Cronbach's alpha coefficient range of $0.66-0.90$ in the subscales of beliefs.

3. Subjective norms. This part is comprised of both "perceived beliefs of significant others' opinions" and "the motivation to comply with significant others' opinions" to provide palliative care. The measure has six items - the influences by spouses, sons, daughters, colleagues, friends, or others - and used a five-point Likert scale from "strongly unaffected" (1) to "strongly affected" (5) [3].

4. Willingness. This section determines the medical directors' consent (yes or no) to provide palliative care services in the community if they encounter an advanced cancer patient. The content of palliative care services included providing consultation, referring patients to palliative care units, home visits, phone follow-ups, and bereavement care for the family.

5. Educational needs. This part identifies the educational needs for providing palliative care: emotional support, communication skills, symptoms management, bereavement support, spiritual care, community resources utilization, principles of palliative care, and legal or ethical issues. The scoring system of this scale is "highly necessary" (5) to "highly unnecessary" (1).

\section{Statistical analysis}

Data management and statistical analysis were performed using SPSS 10.0 statistical software. A frequency distribution was used to describe the demographic data and the distribution of each variable. Mean values and standard deviations were used to analyze the degree of each variable in the knowledge in palliative care, beliefs on palliative care, subjective norm, and educational needs measures. Each item of the above measures, not including the educational needs, was entered into the model as independent variables and the willingness to provide palliative care as the dependent variable. A univariate comparison was carried out to determine the differences of the variables related to willingness. A $p$ value less than 0.05 was considered significant. Then, the backward stepwise logistic regression analysis was carried out to determine the relative values of the variables related to willingness.

\section{Results}

\section{Demographic characteristics}

Table 1 reveals the total number of respondents of 85 . These were mainly $30-49$ years old $(75.5 \%)$ with a mean age of $42.47(\mathrm{SD} \pm 9.95)$ years. More than one third $(32.9 \%)$ of these physicians had no experience in caring for advanced cancer patients, and only $14.1 \%$ had ever referred terminal cancer patients to palliative care. Although more than $80 \%(88.2 \%)$ had received information about palliative care, only $16.5 \%$ could express a clear understanding of that information. Their main sources of information for palliative care were periodicals/books $(45.9 \%)$, in-service education $(35.3 \%)$, or newspapers/magazines $(30.6 \%)$. The

Table 1 Demographic characteristics of the respondent community physicians $(n=85)$

\begin{tabular}{|c|c|}
\hline Variables & Number $(\%)$ \\
\hline \multicolumn{2}{|l|}{ Age (average 42.47 years; $\mathrm{SD}=9.95$ ) } \\
\hline $30-39$ & $40(47.1)$ \\
\hline $40-49$ & $25(29.4)$ \\
\hline$\geq 50$ & $20(23.5)$ \\
\hline \multicolumn{2}{|c|}{ Experience in caring for advanced cancer patients } \\
\hline None & $28(32.9)$ \\
\hline Yes & $57(67.1)$ \\
\hline \multicolumn{2}{|l|}{ Experience in palliative care referral } \\
\hline None & $73(85.9)$ \\
\hline Yes & $12(14.1)$ \\
\hline \multicolumn{2}{|l|}{ Information on palliative care } \\
\hline None & $10(11.8)$ \\
\hline Yes & $75(88.2)$ \\
\hline \multicolumn{2}{|c|}{ Understanding the information about palliative care } \\
\hline Very clearly & $14(16.5)$ \\
\hline A little & $58(68.2)$ \\
\hline Hearing about but do not understand & $13(15.3)$ \\
\hline Never hearing about & $0(0.0)$ \\
\hline \multicolumn{2}{|l|}{ Sources of information (multiple choices) } \\
\hline Periodicals or books & $39(45.9)$ \\
\hline In-service education & $26(35.3)$ \\
\hline Newspapers/magazines & $26(30.6)$ \\
\hline Related seminars & $21(24.7)$ \\
\hline Medical staff & $19(22.4)$ \\
\hline Mass media & $15(17.6)$ \\
\hline Bulletins in hospital & $11(12.9)$ \\
\hline Undergraduate medical education & $7(8.2)$ \\
\hline Palliative training programs & $6(7.1)$ \\
\hline Others & $5(5.9)$ \\
\hline
\end{tabular}


source of knowledge coming from undergraduate medical education was small $(8.2 \%)$.

Variables related to the willingness

to provide palliative care

Table 2 indicates possible factors related to a physician's willingness to provide palliative care, including beliefs, prevailing subjective norms, and knowledge.

The mean score of beliefs toward palliative care was 3.70 (SD 0.38, range 1-5). The four subconcepts of beliefs were: the perception of "threats" caused by the worsening disease process of advanced cancer patients, "benefits of life quality," "benefits of death preparation," and "barriers" in providing palliative care. Their mean scores were 2.81 , $4.29,3.91$, and 3.61, respectively (Table 2). These indicated positive beliefs towards providing palliative care. The higher-score items in perceiving the threat about providing palliative care were: "uncomfortable to meet the advanced cancer patients and to take care of them" (3.91), "influence normal daily medical work and schedule" (3.12), "advanced cancer patient is hopeless for cure" (2.56), and "unable to encounter easily the dying process and the sufferings of dying patients" (2.47) (Table 3 ). The higherscore items in perceiving the barriers toward providing palliative care included: "shorten patient's life, just like euthanasia" (4.24), "no active treatment and only waiting for death" (4.01), and "no aggressive treatment for physical distress" (3.99). On the other hand, the higher-score items in perceiving the benefits about providing palliative care included: "promote life quality and keep the dignity of patients" (4.48), "help the dying have a peaceful death" (4.34), "provide care and companionship" (4.27), and "relieve distress and sufferings" (4.26).

Table 2 Variables related to the willingness of community physicians to provide palliative care $(n=85)$

\begin{tabular}{lcc}
\hline Variables & Mean $( \pm$ SD) & Range \\
\hline $\begin{array}{l}\text { Beliefs: perception of taking care } \\
\text { of advanced cancer patients }\end{array}$ & $3.70(0.38)$ & $1-5$ \\
Factor I: perception of threats & $2.81(0.63)$ & \\
Factor II: perception of benefits & $4.29(0.47)$ & \\
(promote life quality) & & \\
Factor III: perception of benefits & $3.91(0.55)$ & \\
(death preparation) & & \\
Factor IV: perception of barriers & $3.61(0.59)$ & \\
Subjective norms for the provision & $2.83(0.77)$ & $1-5$ \\
of palliative care & & \\
Knowledge of palliative care & $21.72(2.70)$ & $1-28$ \\
Philosophy/principles & $13.08(1.48)$ & $1-14$ \\
Clinical management & $8.64(1.82)$ & $1-14$ \\
\hline
\end{tabular}

Table 3 Beliefs of community physicians regarding palliative care $(n=85)$

Variables

Degree of belief
Mean SD

Factor I: perceived threats from the worsening conditions of advanced cancer patients

1. Uncomfortable to take care of advanced $\quad 3.91 \quad 0.96$ cancer patients

2. Influence normal medical activities $\quad 3.12 \quad 1.16$

3. Hopeless for cure $\quad 2.56 \quad 1.08$

4. Unable to easily face dying process $\quad 2.47 \quad 0.82$ and distress

5. Makes me think about death $\quad 2.40 \quad 1.08$

6. Makes me feel weakness $\quad 2.38 \quad 1.11$

Factor II: perceived benefits for the promotion of life quality

1. Able to promote life quality and keep $\quad 4.48 \quad 0.53$

patient's dignity

2. Enable the patient to die peacefully $\quad 4.34 \quad 0.65$

and have a good death

$\begin{array}{lll}\text { 3. Provide care and companionship } & 4.27 & 0.70\end{array}$

by medical team

4. Relieve pain and other symptoms $\quad 4.26 \quad 0.60$

5. Provide emotional support $\quad 4.26 \quad 0.49$

6. Able to have family support $\quad 4.20 \quad 0.61$

7. Help patient to have a good death $\quad 4.19 \quad 0.55$

Factor III: perceived benefits for better death preparation

1. Respect for patient's religion and $\quad 4.14 \quad 0.64$

burial rites

2. Help to die at home $\quad 4.06 \quad 0.66$

3. Better communication with advanced $\quad 4.05 \quad 0.58$

cancer patients

4. Help medical staff to take care $\quad 3.76 \quad 0.84$

of patients better

5. Avoid the idea of euthanasia $\quad 3.54 \quad 0.91$

Factor IV: perceived barriers to providing palliative care

1. Shorten patient's life just, like euthanasia $\quad 4.24 \quad 0.73$

2. No active treatment and only awaits death $4.04 \quad 0.85$

3. No aggressive treatment for physical $\quad 3.99 \quad 0.81$

symptoms

4. Give up on patients $\quad 3.91 \quad 0.95$

5. Makes patients feel hopeless $\quad 3.85 \quad 0.92$

6. Patients feel abandoned $\quad 3.81 \quad 0.92$

7. Patients have many difficult symptoms $\quad 2.53 \quad 1.03$

$\begin{array}{lll}\text { 8. Makes me feel weakness } & 2.49 & 0.70\end{array}$

\begin{tabular}{lll} 
Total mean & 3.70 & 0.38 \\
\hline
\end{tabular}

Subjective norms for providing palliative care came from the influences of the physicians' significant others. The spouse was the most often cited "significant other" $(44.7 \%)$. But only $52.9 \%$ of the respondents thought that their own significant others knew information regarding palliative care. In addition, about $40 \%$ (45.9\%) of 
Table 4 The willingness of primary care physicians to provide palliative care $(n=85)$

\begin{tabular}{ll}
\hline A willingness to provide palliative care & Number (\%) \\
\hline No & $13(15.3)$ \\
Yes & $72(84.7)$ \\
Provide consultation & $67(93.0)$ \\
Refer to palliative care unit & $63(87.5)$ \\
Provide phone follow-ups & $33(45.8)$ \\
Provide home visits & $29(40.3)$ \\
Provide bereavement care & $21(29.2)$ \\
\hline
\end{tabular}

physicians were "uncertain" whether or not their most significant others would agree with their providing palliative care. However, the total mean of subjective norms was 2.83 (range 1-5), which shows that the physicians were moderately influenced by subjective norms.

With regard to palliative care knowledge, the mean percentage of accurate responses was $74.7 \%$ (SD 13\%). Accurate answers to philosophy/principles and clinical management of palliative care were $93.4 \%$ and $57.3 \%$, respectively. There were eight items, the accuracy of which was less than $60 \%$, including management of pain and dyspnea (four items), emotional support (two items), communicational skills (one item), and insurance policy (one item).

Willingness, and influencing variables related to willingness, to provide palliative care

Nearly $90 \%(84.7 \%)$ of the responding physicians expressed a willingness to provide palliative care in the community if they encountered an advanced cancer patient (Table 4). The majority were willing to provide information about palliative care resources $(93.0 \%)$, refer advanced cancer patients to a palliative care unit $(87.5 \%)$, and conduct a phone follow-up or monitoring (45.8\%). However, the willingness to conduct home visits and to provide bereavement care was seen less $(40.3 \%$ and $29.2 \%$, respectively). With regard to the related variables, only the item whether having 5 years of clinical practice had significant differences in the willingness to provide palliative care $\left(\chi^{2}=8.66, p<0.01\right)$ in univariate analysis.

Further analyzing home visitation, although all in the willing group had higher mean values than those in the unwilling group in terms of knowledge and subscales of beliefs to palliative care, there was no item that had any statistically significant difference in the willingness to provide home visits in the univariate analysis. Afterward, the study still performed a stepwise logistic regression analysis to determine the relative values of the variables related to willingness. The results reveal that only the subjective norms remained in the model $(\mathrm{OR}=1.88,95 \% \mathrm{CI}=1.17-3.01$, $p<0.05$ ). However, the constant (other possible variables not entered in the study) in the model also showed the significant influences $(p<0.01)$ to the willingness of providing home visiting. These results implied that some other important variables related to the physicians' willingness were not included in the analysis in our study, which need further study.

Educational needs of community physicians to provide palliative care

As shown in Table 5, the fields in which respondents perceived their educational needs were those related to "emotional support" (mean=4.28, range 1-5) and "communication skills" (mean=4.26). These were followed by "bereavement support of families" (mean=4.14) and "how to manage physical symptoms" (mean=4.13). Concerning symptom management, pain $(100.0 \%)$, dyspnea $(94.4 \%)$, and bed sores $(90.1 \%)$ were the areas in most need of training of physicians in providing palliative care in their communities.

Table 5 Educational needs for palliative care in the community $(n=85)$

\begin{tabular}{|c|c|c|c|c|c|c|}
\hline Variables & Highly necessary & Necessary & Fair & Unnecessary & Mean score & SD \\
\hline Method of emotional support & $33(38.8)$ & $44(51.8)$ & $7(8.2)$ & $1(1.2)$ & 4.28 & 0.67 \\
\hline Skills to communicate with advanced cancer patients & $31(36.5)$ & $45(52.9)$ & $9(10.6)$ & $0(0.0)$ & 4.26 & 0.64 \\
\hline Bereavement support & $26(30.6)$ & $46(54.1)$ & $12(14.1)$ & $1(1.2)$ & 4.14 & 0.69 \\
\hline Management of physical symptoms & $18(21.2)$ & $51(72.9)$ & $5(7.1)$ & $0(0.0)$ & 4.13 & 0.53 \\
\hline Spiritual care & $27(31.8)$ & $43(50.6)$ & $14(16.5)$ & $1(1.4)$ & 4.13 & 0.72 \\
\hline Community resources utilization & $21(24.7)$ & $51(60.0)$ & $13(15.3)$ & $0(0.0)$ & 4.09 & 0.63 \\
\hline Content of palliative care & $20(21.4)$ & $51(60.0)$ & $11(12.9)$ & $3(3.5)$ & 4.04 & 0.72 \\
\hline Ethical/legal issues & $18(20.0)$ & $50(58.8)$ & $15(17.6)$ & $2(2.4)$ & 3.99 & 0.70 \\
\hline Total mean score & & & & & 4.13 & 0.48 \\
\hline
\end{tabular}

( ) Percentages 


\section{Discussion}

To our knowledge, this study is one of the first to investigate the willingness of community physicians to provide palliative care, particularly in the Asia Pacific region. Our response rate of $62.8 \%$ was only similar to the mean response rate of $61 \%$ in published general practitioner studies [26]. The response rate may have been affected by the community physicians' heavy workload at the time of the study, by the volume or content of questionnaires they received, or even because of divergent views on participating. Although the moderate response rate limits the strength of any conclusion, it can still reflect views of community physicians and provide some important information for us.

The study focused on the rural areas, which usually give the impression of being more supportive and having a greater possibility for providing a nice home environment for terminal care despite the possible absence of medical facilities. The national generalizability of the study is clear since our study sample includeed all medical directors of health stations in most of the rural communities, covering more than $70 \%$ of Taiwan's geographic area.

Community physicians are the key professionals most in touch with patients' medical needs in the rural community. The responsibilities of physicians in health stations in Taiwan can be recognized in providing comprehensive medical care for patients in their communities and in liaising with other specialist colleagues. Therefore, the study physicians have the potential to play an important role in the care of the dying in their communities, and hence, the main reason for our study was the investigation as to their willingness. Meanwhile, interest is increasing in other countries in the provision of community palliative care for advanced cancer patients [21]. Most countries in the Asia Pacific region, such as Japan, Korea, Mainland China, and Thailand, have also developed an organized community health care system where the community medical teams are accountable for the health of people in a designated geographical district and may have the same background to develop community palliative care.

Physicians in government health stations in Taiwan, requiring a minimum 2 years of postgraduate training, are conventionally trained in providing primary medical care, public health services, disease prevention, and health promotion but not in providing palliative care. Their focus is the care of common health problems of community inhabitants. Rarely do they provide care for advanced cancer patients and thus have less opportunity to gain experience in providing palliative care, and therefore risk losing both experience and confidence. This vicious cycle exists in community care. About one third of respondents (32.9\%) expressed that they had never encountered the care problems of advanced cancer patients in their work experiences. Although nearly $90 \%(84.7 \%)$ of the community physicians in this study were willing to provide palliative care when they had to, they would find this difficult in rural communities because it is beyond their expertise. We can recognize this in the findings from the community physicians surveyed: insufficient palliative care knowledge - especially in clinical practice in managing common problems of advanced cancer patients such as pain, dyspnea, and anorexia - and feelings of threat or barriers from the worsening conditions of advanced cancer patients or difficulty in communicating with these patients. These factors might affect their willingness to directly take care of advanced cancer patients. Therefore, they would be likely limit their services to providing consultation $(93.0 \%)$ and making referrals $(87.5 \%)$, but would be less likely to provide home visits $(40.3 \%)$ or bereavement support for the family $(29.2 \%)[11,20]$.

As regards the beliefs of providing palliative care, the major reason related to the threat perceived by the physicians in taking care of advanced cancer patients was "uncomfortable to meet with the advanced cancer patients and to take care of them." It indicated the difficulties for these physicians in communicating with the advanced cancer patients or providing support. In Taiwan, the traditional medical education has mainly focused on the cure of diseases rather than the relief of suffering or the comfort of patients, which makes the physician who treats a patient whose disease becomes incurable a "medical failure." Lack of education or training in providing care for advanced cancer patients both in undergraduate and postgraduate periods inevitably influences the ability and the positive attitude of physicians in meeting terminal patients. This result is compatible with the findings of educational needs, which indicated "the method to give emotional support" and "the technique to communicate with patients" as the two educational priorities. Concerning the barriers perceived by physicians in providing palliative care, it is surprising that the main reasons were "palliative care may shorten a patient's life, just like euthanasia" and "palliative care gives up on active treatments and only awaits death." These surprising findings point to the lack of education and training in the real content and ethical nature of palliative care. Despite the physicians' recognition that palliative care can promote the best life quality for patients, they could not differentiate clearly between palliative care and euthanasia. Education toward this ethical issue will remain a worthwhile effort.

The other important variable related to the threat perceived by the study physicians in taking care the advanced cancer patients was "influence normal daily medical activities." This finding indicates that the palliative care service has not yet been included in the essential part of community health services. Taking care of the advanced cancer patient usually requires much time. Hence, beside the support of governmental policy, the practical work to enhance community palliative care is to extend adequate insurance payment for this time-consuming work.

With regard to home visits, previous studies reported many advanced cancer patients feel they are not visited 
frequently enough. They rate the quality of home palliative care in terms of home visiting patterns. Nevertheless, the number of home visits, especially for the dying, continues to fall $[4,17,28]$. Comparing the other three factors in the beliefs measure, the mean score of "perceived benefits to provide palliative care" was the highest. It seems reasonable to suppose that community physicians' willingness to aactively provide home palliative care is optimistic in Taiwan if there is insurance coverage and full governmental support.

To provide more productive palliative care to the community, developing a better health care system in the community is fundamental. In Taiwan, beside well-organized government health stations, training programs in family and community medicine have been advocated for 20 years and are now available to most community physicians, community nurses, and other health professionals. On this basis, constructing a primary care team comprised of primary care physicians, nurses, and other health professionals, and improving their team dynamics for coordinating community resources and providing palliative care, will be a strong first step. Providing services such as "consultations, referrals, and phone visits" would be practical in the initial stage. Otherwise, a referral system and communication network should be established among government health stations, palliative care units, and home care teams. Enhancing discharge planning for advanced cancer inpatients is also important.

The findings of stepwise logistic regression analysis to the related variables imply that some important variances might not be included in the empirical tests in our study, such as the personality of respondents, referral system, health care manpower, health insurance, health policy, and social welfare system [22]. These could be influential and should be investigated in further studies.

In conclusion, making efforts in three areas is key for the community palliative care movement in Taiwan. These key areas are: (1) effective education on practical knowledge of palliative care for community physicians, (2) incorporation of palliative care into the medical education, particularly in terms of communication skills and ethical roles, and (3) active health policy administration, including adequate insurance payment and a sound referral system.

Acknowledgements This study was supported by the National Science Council of Taiwan. The authors are grateful to the 85 medical directors of the governmental health stations for their participation. We also acknowledge Ms. K.H. Chao, C.W. Liu, and Y.C. Chen for their assistance in preparing the manuscript.

\section{Appendix A}

1. Pain and other physical symptoms in advanced cancer patients can be mostly controlled by good palliative care.
2. The goal of palliative care is to achieve the best possible quality of life for the patients and the families, not to cure.

3. Palliative care is respect natural death, not to shorten the life of patients.

4. Psychological, social, and spiritual problems are paramount to the palliative care team who give appropriate consultation and management.

5. Allocation of community resources for patients and families is also the content of palliative care.

6. Palliative care is a work of interdisciplinary fields.

7. It is not the content of palliative care to provide the family care for advanced cancer patients.

8. The palliative care team provides bereavement support for the family after the patient's death.

9. It is the content of palliative care to help patients face and prepare for death.

10. Palliative care respects for the autonomy and individualized needs of patients.

11. Palliative care provides spiritual care for advanced cancer patients.

12. The staff of the palliative care unit are required to receive adequate palliative care professional training.

13. Palliative care is an alternative for advanced cancer patients.

14. Suffering and physical pain are synonymous.

15. The palliative care team actively controls the pain and other physical symptoms of advanced cancer patients.

16. Palliative care services in Taiwan include inpatient care and home care.

17. The cost of palliative care services in Taiwan has been included in the National Health Care Insurance System.

18. Adjuvant therapies are important in managing pain.

19. Drug addiction is a major problem when morphine is used on a long-term basis for the management of pain.

20. Individuals who are taking morphine should also follow a bowel regimen.

21. The provision of palliative care requires emotional detachment.

22. During the terminal stages of an illness, drugs that can cause respiratory depression are appropriate for the treatment of severe dyspnea.

23. Men generally reconcile their grief more quickly than women.

24. The use of placebos is appropriate in the treatment of some types of pain.

25. Demerol is not an effective analgesic for the control of chronic pain.

26. Manifestations of chronic pain are different from those of acute pain.

27. The loss of a distant or contentious relationship is easier to resolve than the loss of one that is close or intimate.

28. Pain threshold is lowered by fatigue or anxiety. 


\section{Appendix B}

Circle the number that represents your OPINION (there are no right or wrong answers)

$5=$ strongly agree, 4=agree, $3=$ uncertain, $2=$ disagree, 1=strongly disagree:

Perception of the threats from the worsening conditions of advanced cancer patients is:

1. Uncomfortable to take care of advanced cancer patients

2. Influence normal medical activities

3. Hopeless for the cure

4. Unable to easily face dying process and distress

5. Makes me think about death

6. Makes me feel weakness

Perceptions of the benefits for the promotion of life quality is:

7. Able to promote life quality and keep the dignity

8. Able to die peacefully and have good death

9. Having care and accompanied by medical team

10. Relief pain and other symptoms
11. Emotional support

12. Able to have family support

13. Help to have good death

Perceptions of the benefits for better death preparation is:

14. Respect for patient's religion and burial rites

15. Help to die at home

16. Better communication with advanced cancer patients

17. Help medical staff to take care of patients better

18. Avoid the idea of euthanasia

Perception of the barriers to provide palliative care is:

19. Shorten patient's, life just like euthanasia

20. No active treatment and only awaits death

21. No aggressive treatment for physical symptoms

22. Give up patients

23. Make patients feel hopeless

24. Patients feel abandoned

25. Advanced cancer patients have many difficult symptoms

26. Make me feel weak to the life

\section{References}

1. Addington-Hall JM, MacDonald LD, Anderson HR et al (1991) Dying from cancer: the views of bereaved family and friends about the experiences of terminally ill patients. Palliat Med 5:207-214

2. Boyd KJ (1993) Palliative care in the community: views of general practitioners and community physicians in east London. J Palliat Care 9:33-37

3. Carter WB (1990) Health behavior as a rational process: theory of reasoned action and multi-attribute utility theory. In: Glanz K, Lewis FM, Rimes BK (eds) Health behavior and health education. Jossey-Bass, San Francisco, pp 39-62

4. Cartwright A (1990) The role of the general practitioner in caring for people in the last year of their lives. King Edward's Hospital Fund Report

5. Catalán Fernández JG, Pons Sureda O, Recober Martinz A et al (1991) Dying of cancer: the place of death and family circumstances. Med Care 29:841-852

6. Charlton RC (1991) Attitudes towards care of the dying. A questionnaire survey of general practice at tenders. Fam Pract 8:356-359
7. Chiu TY, Ohi G (1995) The attitudes toward terminal care in rural communities between Taiwan and Japan: a comparative study. Unpublished master's thesis. University of Tokyo, Tokyo, Japan

8. Chiu TY (1996) Annual Report of Palliative Care of National Taiwan University Hospital. National Taiwan University Hospital, Taipei

9. Department of Health, The Executive Yuan (1989) National Project of the Department of Health-Medical Care Network System in Taiwan. J Nurs 36:5-11 (in Chinese)

10. Department of Health, The Executive Yuan (2004) Vital statistics, Republic of China, 2003

11. Hanratty B (2000) Palliative care provided by GPs: the carer's viewpoint. $\mathrm{Br}$ J Gen Pract 50:653-654

12. Hearn J, Higginson IJ (1998) Do specialist palliative care teams improve outcomes for cancer patients? A systematic literature review. Palliat Med 12:317-332

13. Higginson IJ, Edmons P (1999) Services, costs and appropriate outcome in end of life care. Ann Oncol 10:135-136

14. Hinton J (1994) Can home care maintain an acceptable quality of life for patients with terminal cancer and their relatives? Palliat Med 8:183-196
15. Hu WY, Chiu TY, Chuang RB et al (1999) The needs and satisfaction of main family caregivers in domiciliary palliative care. Formos J Med 3:527537 (in Chinese)

16. Hu WY, Chiu TY, Cheng YR et al (2004) Why Taiwanese hospice patients want to stay in hospital: health-care professionals' beliefs and solutions. Support Care Cancer 12:285-292

17. Isaacs B (1971) The concept of predeath. Lancet 1:1115-1119

18. Kai I, Ohi G, Yano E et al (1993) Communication between patients and physicians about terminal care: a survey in Japan. Soc Sci Med 36:11511159

19. Lan CF, Hsiung HY (1993) Progress and issues in the long-term care for the elderly in Taiwan. J Nurs 40:15-24 (in Chinese)

20. Lloyd-Williams M, Wilkinson C, Lloyd-Williams F (2000) General practitioners in North Wales: current experiences of palliative care. Eur J Cancer 9:138-143

21. Low JA, Liu RK. Strutt R et al (2001) Specialist community palliative care services - a survey of general practitioners' experience in Eastern Sydney. Support Care Cancer 9:474-476 
22. McRae S, Caty S, Nelder $M$ et al (2000) Palliative care on Manitoulin Island. View of family caregivers in remote communities. Can Fam Physician 46:1301-1307

23. Nielsen BB, McMillillan S, Diaz E (1992) Instruments that measure beliefs about cancer from a cultural perspective. Cancer Nurs 15:109-115
24. Oneschuk D, Fainsinger R, Hanson J et al (1997) Assessment and knowledge in palliative care in second year family medicine residents. J Pain Symptom Manage 14:265-273

25. Ross MM, McDonald B, McGuinness J (1996) The palliative care quiz for nursing (PCQN): the development of an instrument to measure nurses' knowledge of palliative care. J Adv Nurs 23:126-137

26. Sibbald B, Addington-Hall JM, Brenneman D et al (1994) Telephone versus postal surveys of general practitioners, methodological consideration. Br J Gen Pract 44:297-300
27. Townsend J, Frank AO, Fermant D (1990) Terminal cancer care and patients' preference for place of death: a prospective study. BMJ 301:415-417

28. Williams EL, Fitton F (1990) General practitioner response to elderly patients discharged from hospital. BMJ 300:159-161 